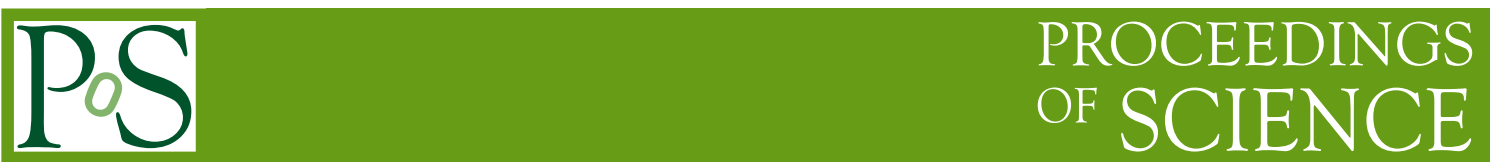

\title{
Combined Higgs boson measurements at the ATLAS experiment
}

\section{Matthew H. Klein ${ }^{a, *}$, on behalf of the ATLAS Collaboration \\ ${ }^{a}$ University of Michigan}

E-mail: matthew.henry.klein@cern.ch

\begin{abstract}
Higgs boson measurements performed by ATLAS with data collected in LHC Run 2 have been combined, resulting in a precise set of measurements of Higgs boson properties. Results are found to be consistent with the expectations of the Standard Model, and limits are set in a variety of BSM scenarios. These results extend those from an earlier Higgs boson combination, which did not include data collected in 2018, to use the full LHC Run 2 dataset for the $H \rightarrow \gamma \gamma, H \rightarrow Z Z \rightarrow 4 l$, $H \rightarrow \mu \mu, V(l e p) H \rightarrow b b$, and VBF $H \rightarrow i n v$ production modes and decays.
\end{abstract}

40th International Conference on High Energy physics - ICHEP2020

July 28 - August 6, 2020

Prague, Czech Republic (virtual meeting)

${ }^{*}$ Speaker 
Many separate ATLAS [1] Higgs boson measurements have been previously performed for different production modes and Higgs boson decays. The measurements frequently measure quantities that are independent of the Higgs boson decay, such as the cross-sections for the different production modes or differential measurements of Higgs boson kinematics. A combination of previous Higgs boson measurements has been performed using data collected in LHC Run 2, in order to produce the most precise set of Higgs boson cross-section measurements by ATLAS, as well as to set limits on various BSM effects and models [2].

Prior to this result, the most recent ATLAS Higgs boson combination was performed using data collected between 2015 and 2017 [3], while for this result, several individual measurements have been updated to the full Run 2 dataset. The full Run 2 dataset is included for $H \rightarrow \gamma \gamma$ [4], $H \rightarrow Z Z \rightarrow 4 l$ [5], $H \rightarrow \mu \mu$ [6], V(lep) $H \rightarrow b b$ [7], and VBF $H \rightarrow$ inv [8]. Only 2015-2016 data is included for $H \rightarrow \tau \tau$ [9], $H \rightarrow W W$ [10], $\mathrm{ttH}$ (multilepton) [11, 12], $\mathrm{ttH}$ (bb) [11-13], and VBF $H \rightarrow b b[14]$.

\section{Production Cross-section}

Floating a single global signal strength, the Higgs boson cross-section relative to the Standard Model expectation is measured to be

$$
\mu=1.06 \pm 0.07=1.06 \pm 0.04 \text { (stat.) } \pm 0.03 \text { (exp.) }{ }_{-0.04}^{+0.05} \text { (sig. th.) } \pm 0.02 \text { (bkg. th.) }
$$

Compared to earlier measurements, largely due to the increased statistics of the dataset, the measurement has reached the point where the systematic uncertainties are dominant relative to the statistical uncertainties. Further improvement in the measurement of the Higgs boson production cross-section will require improvements in Higgs boson modeling.

The second measurement is of the production cross-sections for each of the five major Higgs boson production modes. The Higgs boson branching fractions are fixed to their SM expectations, and the production cross-sections are floated. The results of this measurement are shown in Figure 1. The precision of the measurement of gluon fusion production $(\mathrm{ggF})$ is comparable to the theory

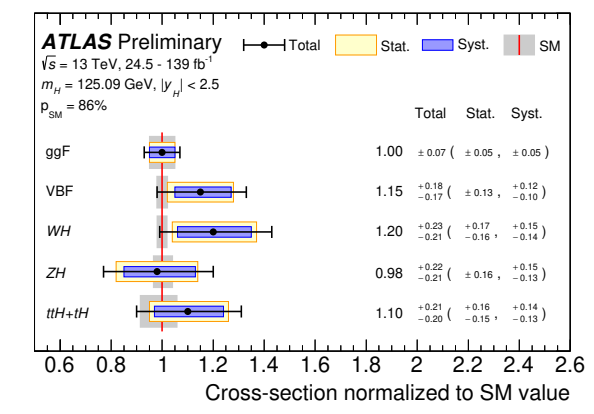

Figure 1: Signal strength measurement for each of the major Higgs boson production modes. Results are obtained from a simultaneous fit, floating each signal strength parameter [3].

uncertainty. Additionally, for all five production modes, the measurements correspond to greater than $5 \sigma$ with respect to the SM background expectation. 


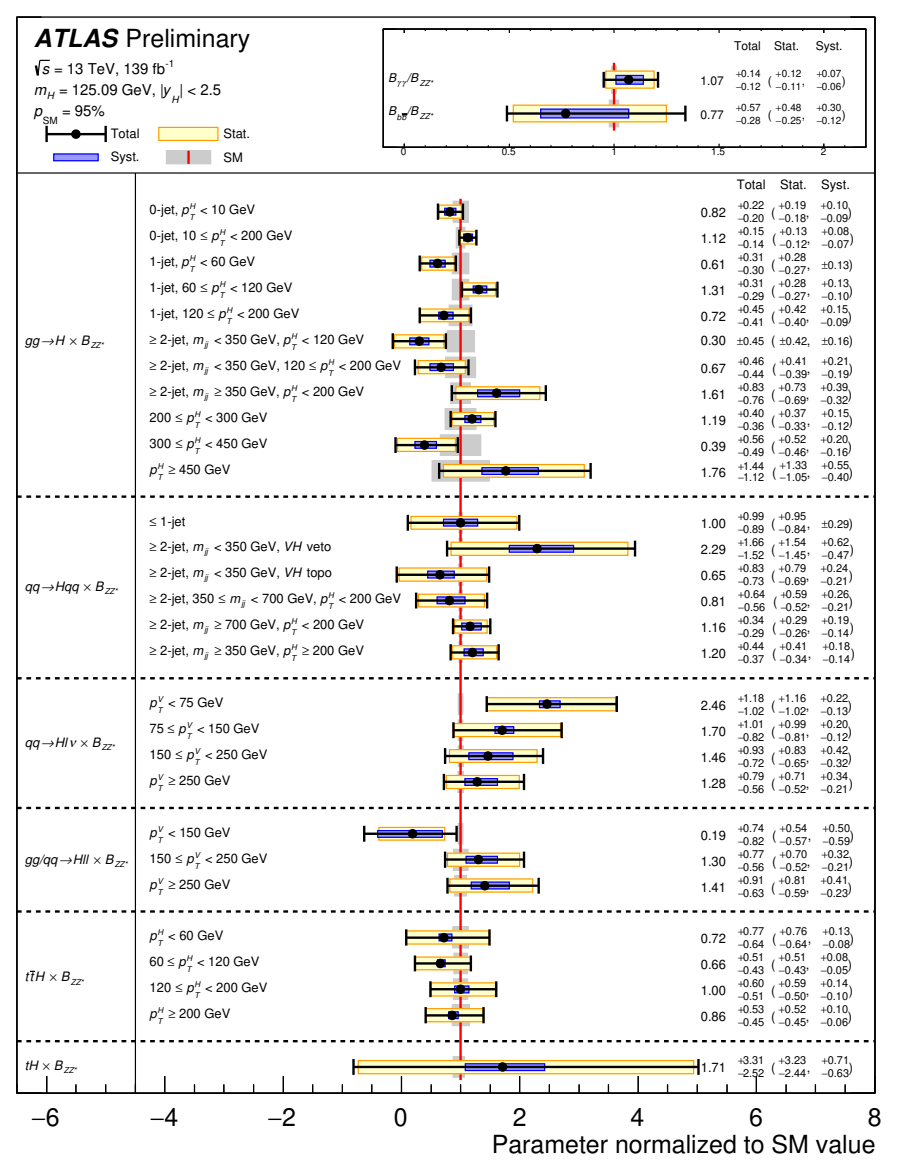

Figure 2: Results for STXS measurements for all the bins considered in this combination [3].

For the third measurement, the signal strength is measured for each combination of production mode and Higgs boson decay. The results are found to be consistent with each other and with the SM expectation.

\section{Simplified Template Cross Section}

The next measurement is of the Simplified Template Cross Sections (STXS) [15]. The Higgs boson phase space is partitioned into a set of non-overlapping regions. These regions are chosen to be sensitive to deviations from the SM, to avoid large theory uncertainties, and to approximately match experimental selections so as to minimize model-dependent extrapolations. Additionally, bins are merged in order to avoid large anti-correlations between bins not separately constrained from measurements. For this measurement, only $H \rightarrow \gamma \gamma, H \rightarrow Z Z \rightarrow 4 l$, and $V(l e p) H \rightarrow b b$ are considered.

The results are shown in Figure 2. Measurements are found to agree well with the SM expectation, albeit with large uncertainties in many bins. For tH production, an upper limit of 8.4 times the SM expectation is observed, compared to an expectation of 8.2 times the SM expectation. 


\section{3. $\kappa$ Measurements}

The same particle-level Higgs boson couplings can appear in both the Higgs boson production and decay processes. For example, ggF production and the $H \rightarrow \gamma \gamma$ processes both largely proceed through top loop diagrams, and deviations of the Higgs boson coupling to top quarks will affect both processes. For the next set of measurements, modifiers $\kappa$ are defined for the couplings of the Higgs boson to SM particles, either using effective couplings for loops or using particle-level coupling modifiers [16].

For the first of these measurements, a coupling modifier is floated for each of $\mathrm{W}, \mathrm{Z}, \mathrm{t}, \mathrm{b}, \tau$, and $\mu$. The result of this measurement is shown in Figure 3. Excellent agreement of couplings is found through three orders of magnitude of particle masses.

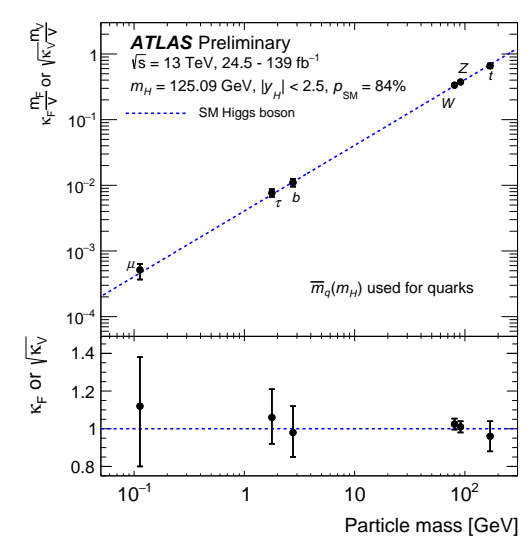

Figure 3: Measurement of each $\kappa$ parameter, in a fit that does not include BSM couplings or modifications to effective couplings [3].

Effective couplings $\kappa_{g}$ and $\kappa_{\gamma}$ are sensitive to new particles and BSM effects appearing in loops. For the next measurement, these effective couplings are floated, either fixing or floating the branching fraction to invisible particles $B_{i n v}$ and undetected decays $B_{\text {undet }}$. $B_{i n v}$ is also constrained from the VBF $H \rightarrow i n v$ measurement. The results of this measurement are shown in Figure 4a. Good agreement is observed with the SM expectation. Next, extra coupling modifiers are introduced, in addition to the effective couplings for loop processes. The results of this measurement are shown in Figure $4 \mathrm{c}$, where good agreement with the SM is observed. Negative $\kappa_{t}$ is excluded at $2.9 \sigma$, with an expected exclusion at $2.7 \sigma$.

The previously described measurements all rely on assumptions on the Higgs boson total width. In order to make a more model-independent measurement, the ratios of $\kappa$ parameters are measured $(\lambda)$, such that the dependence on the Higgs boson width cancels. These ratios can be sensitive to a wide range of BSM effects. For example, $\lambda_{t g}$ is sensitive to new colored particles entering into the effective ggH coupling, and $\lambda_{\gamma Z}$ is sensitive to the contribution of new charged particles. The results of these measurements are given in Figure $4 b$. 


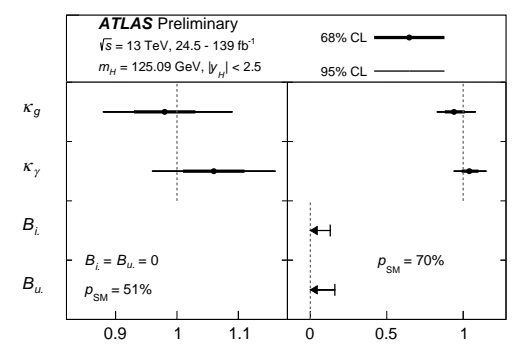

(a)

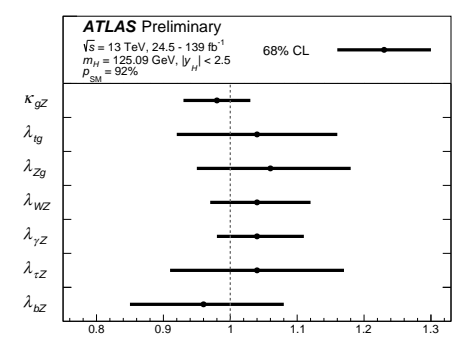

(b)

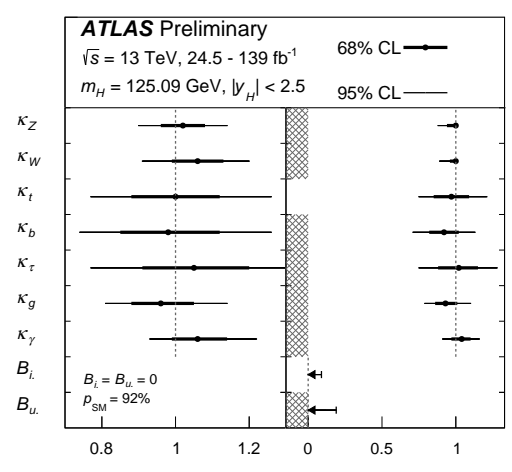

(c)

Figure 4: Measurement of $\kappa$ parameters for three different setups, in which 4 a only the effective couplings are floated, $4 \mathrm{~b}$ the effective and direct couplings are floated, and $4 \mathrm{c}$ the ratios of couplings are floated [3].

\section{2HDM Interpretation}

Lastly, the Higgs boson measurements are interpreted in the context of the 2HDM [16]. Example results of these interpretations are shown in Figure 5 [17, 18]. In the first example, corresponding to the Type-I 2HDM, one Higgs doublet couples to vector bosons, while the other couples to fermions. In the second example, corresponding to the Type-II 2HDM, one Higgs doublet couples to up-type quarks, and the other couples to down-type quarks and charged leptons.

\section{Summary}

Various Higgs boson measurements performed by the ATLAS Collaboration using data collected in LHC Run 2 have been combined to produce a precise set of measurements of Higgs boson properties. Results are found to be consistent with the SM expectations, and limits have been set on BSM scenarios.

\section{References}

[1] ATLAS Collaboration, JINST 3 (2008) S08003.

[2] ATLAS Collaboration, ATLAS-CONF-2020-027, 2020. 


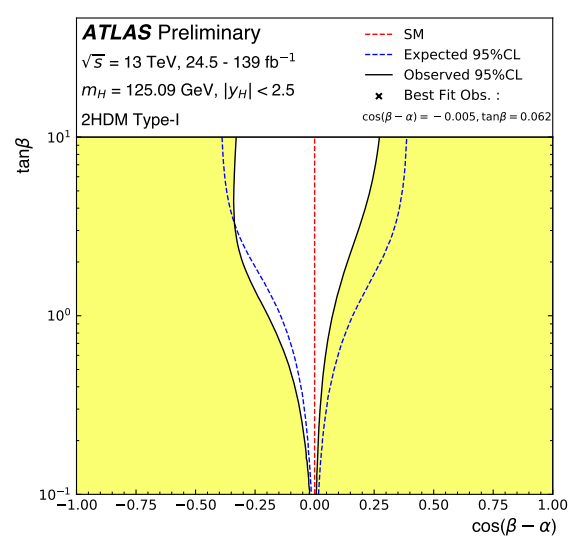

(a)

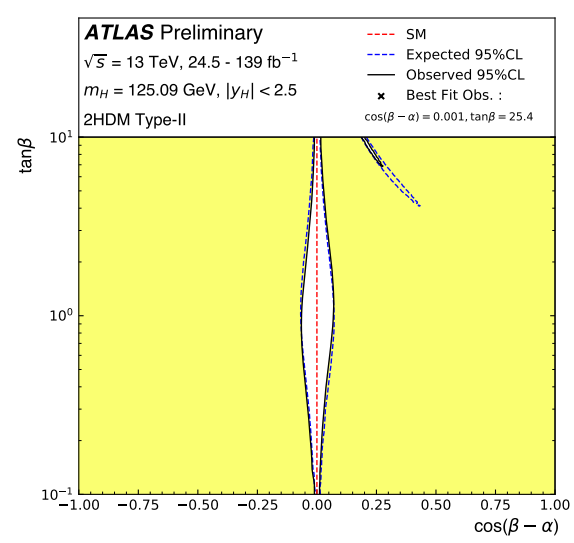

(b)

Figure 5: Interpretation for 2HDM scenarios, showing the region excluded by this measurement [3].

[3] ATLAS Collaboration, Phys. Rev. D 101 (2020) 012002 [1909.02845].

[4] ATLAS Collaboration, ATLAS-CONF-2020-026, 2020.

[5] ATLAS Collaboration, Eur. Phys. J. C 80 (2020) 957 [2004 . 03447].

[6] ATLAS Collaboration, Submitted to Phys. Lett. B (2020) [2007.07830].

[7] ATLAS Collaboration, Submitted to EPJC (2020) [2007. 02873].

[8] ATLAS Collaboration, ATLAS-CONF-2020-008, 2020.

[9] ATLAS Collaboration, Phys. Rev. D 99 (2019) 072001 [1811. 08856].

[10] ATLAS Collaboration, Phys. Lett. B 789 (2019) 508 [1808.09054].

[11] ATLAS Collaboration, Phys. Rev. D 97 (2018) 072003 [1712. 08891].

[12] ATLAS Collaboration, Phys. Lett. B 784 (2018) 173 [1806.00425].

[13] ATLAS Collaboration, Phys. Rev. D 97 (2018) 072016 [1712 .08895].

[14] ATLAS Collaboration, Phys. Rev. D 98 (2018) 052003 [1807.08639].

[15] N. Berger et al., 1906.02754.

[16] LHC Higgs Cross Section Working Group, S. Heinemeyer et al., CERN-2013-004 (2013) [1307.1347].

[17] S. L. Glashow and S. Weinberg, Phys. Rev. D 15 (1977) 1958.

[18] E. A. Paschos, Phys. Rev. D 15 (1977) 1966. 